\title{
A Quantized Surprise from Fermi Surface Topology
}

\section{The quantized conductance of a two-dimensional electron gas can reflect its Fermi surface topology.}

By Martin Rodriguez-Vega

S ome materials possess topological contributions to their conductance and resistance that are resilient against imperfections in their structure. The origin of these contributions is rooted in "topological numbers" that characterize the ground state of the electrons in a material. In metals, the electrons responsible for conductance and resistance are housed on the material's Fermi surface, which can also be characterized by a topological number. But, until now, experimentalists did not know how to measure this parameter directly. Now, Charles Kane of the University of Pennsylvania has theoretically found a way to access experimentally the Fermi surface topological number [1]. His work shows that the nonlinear conductance of a two-dimensional electron gas should be quantized because of its Fermi surface topology.

Kane considered a two-dimensional electron gas to which were attached three metallic leads. He then carried out the following thought experiment. First, he visualized dividing the plane on which the leads sat into three regions, one for each lead. Then, he imagined applying separate voltage pulses to two regions

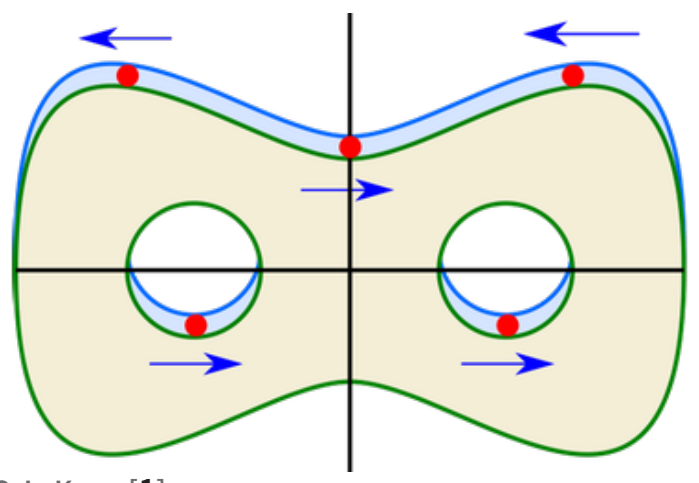

with strengths such that they accelerated the Fermi surface electrons toward the third region. He then considered what happened to the number of electrons in that third region.

Kane's thought experiment showed that the number of electrons in the third region was dependent on the Fermi surface topology of the system. He found the same result more rigorously via a quantum nonlinear response theory calculation.

Experimental verification of this prediction should be possible, but it will require solving challenges related to sample design and to measuring the scattering properties of the accelerated electrons. For now, the application of this predicted quantized conductance remains elusive. But, Kane says, the result indicates that two-dimensional electrons still hold topological surprises.

Martin Rodriguez-Vega is an Associate Editor for Physical Review Letters.

\section{REFERENCES}

1. C. L. Kane, "Quantized nonlinear conductance in ballistic metals," Phys. Rev. Lett. 128, 076801 (2022).

Credit: C. L. Kane [1] 